\title{
Efficacy and implications of a 48-h cutoff for video capsule endoscopy application in overt obscure gastrointestinal bleeding
}

Authors

Institution
Seung Han Kim*, Bora Keum*, Hoon Jai Chun, In Kyung Yoo, Jae Min Lee, Jong Soo Lee, Seung Joo Nam, Hyuk Soon Choi, Eun Sun Kim, Yeon Seok Seo, Yoon Tae Jeen, Hong Sik Lee, Soon Ho Um, Chang Duck Kim

Division of Gastroenterology and Hepatology, Department of Internal Medicine, Institute of Digestive Disease and Nutrition, Korea University College of Medicine, Seoul, Republic of Korea submitted

3. December 2014 accepted after revision 23. February 2015

\section{Bibliography}

DOI http://dx.doi.org/

10.1055/s-0034-1391852

Published online: 5.5.2015

Endosc Int Open 2015; 03:

E334-E338

(c) Georg Thieme Verlag KG

Stuttgart · New York

E-ISSN 2196-9736

Corresponding author

Hoon Jai Chun, MD PhD

Division of Gastroenterology and Hepatology

Department of Internal

Medicine

Institute of Digestive Disease and Nutrition

Korea University College of Medicine

126-1, Anam-dong 5 ga

Seongbuk-gu

Republic of Korea

Fax: +82-2-9531943

drchunhj@chol.com
Seoul 136-705

Background and study aims: Early video capsule endoscopy (VCE) may provide a high diagnostic yield and improve clinical outcomes in patients with overt obscure gastrointestinal bleeding (OGIB); however, there is no practical recommendation for the ideal timing of VCE application in overt OGIB. Therefore, this study investigated the diagnostic yield and efficacy of VCE to assess overt OGIB with respect to the timing of application. Patients and methods: We retrospectively enrolled patients who had undergone VCE for overt OGIB between April 2004 and February 2014 at a tertiary referral academic center. We included hemodynamically stable patients who underwent VCE for overt OGIB after negative bidirectional endoscopy. We analyzed the diagnostic yield of VCE, therapeutic intervention rate, and length of hospital stay.

\section{Introduction}

$\nabla$

Obscure gastrointestinal bleeding (OGIB) is defined as gastrointestinal bleeding of unknown origin that persists or recurs despite a negative finding from an initial bidirectional endoscopy [1]. OGIB accounts for approximately $5 \%$ of all cases of gastrointestinal bleeding [1,2]. Overt OGIB presents with proof of obvious bleeding (i.e. melena or hematochezia).

Early deployment of video capsule endoscopy (VCE) close to the occurrence of bleeding may improve diagnostic yield in OGIB patients $[3,4]$. However, the optimal timing of VCE application in patients with overt OGIB is still unclear, and limited data are available concerning the usual history of overt OGIB, including early diagnosis, proper management, and clinical outcomes [5]. More information is needed to perform VCE appropriately. Moreover, few studies have investigated only overt OGIB, and no data are available

* These authors contributed equally to this work.
Results: A total of 94 patients underwent VCE to assess overt OGIB. The diagnostic yields in the groups that underwent VCE $<48 \mathrm{~h}$ and $>48 \mathrm{~h}$ from the last overt OGIB were $66.7 \%$ and $40.6 \%$, respectively $(P=0.019)$. Therapeutic intervention was performed in $26.7 \%$ and $9.4 \%$ of patients in the $<48$-h and $>48$-h groups, respectively $(P=$ 0.028 ). The mean lengths of hospital stay in the $<48$-h and $>48$-h groups were 5 days (95\% confidence interval $[\mathrm{CI}], 4.8-7.7)$ and 7 days $(95 \% \mathrm{CI}$, 6.9 -10.1), respectively $(P=0.039)$.

Conclusions: Performing VCE within 2 days from the last overt OGIB results in a higher diagnostic yield, higher therapeutic intervention rate, and shorter hospital stay. Therefore, VCE application with a 48-h cutoff could improve the outcome of patients with overt OGIB.

with regard to the superiority of VCE performed within 2 days of the last OGIB with respect to diagnostic yield and clinical outcomes.

Therefore, this study primarily aimed to demonstrate the high diagnostic yield of VCE performed within 48 hours after the last overt OGIB. The secondary objective was to assess its benefits with respect to therapeutic intervention rate and length of hospital stay.

A previous series showed that OGIB lesions are found mostly in the small bowel [6]. VCE is an invaluable modality for investigating the small bowel. It enables the exploration of the whole small bowel in about $85 \%$ of patients [7], and its diagnostic yield ranges from $38 \%$ to $83 \%$ [8]. Therefore, VCE is a crucial diagnostic method for OGIB [9-11]. Earlier studies demonstrated that VCE has a higher diagnostic yield when investigating OGIB than other endoscopic and radiological modalities $[10,12]$. The early precise diagnosis of overt OGIB can lead to the administration of an appropriate specific intervention, better outcomes, and reduce unnecessary medical inter- 


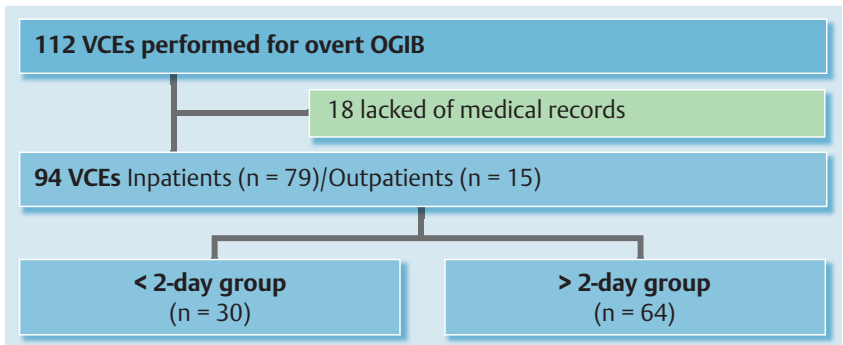

Fig. 1 Study design flow chart. VCE, video capsule endoscopy; OGIB, obscure gastrointestinal bleeding.

ventions and financial expense from other diagnostic modalities and procedures required to define the exact bleeding lesion. Although some studies report better outcomes from the early deployment of VCE in overt OGIB patients [13-15], the optimal timing of VCE application is unclear, particularly in patients with obvious bleeding but with hemodynamic stability.

\section{Patients and methods}

\section{Patients}

The subjects of this study were patients with overt OGIB who underwent VCE to determine the bleeding source. We conducted a single-center retrospective study at the Korea University Medical Center Anam Hospital enrolling patients who had undergone VCE for overt OGIB between April 2004 and February 2014. VCE was performed on 509 patients during this period. After excluding VCEs performed for other symptoms such as anemia and recurrent diarrhea as well as the evaluation of abdominal pain, 94 VCE $(18 \%)$ procedures performed for overt OGIB in patients with negative bidirectional endoscopy and hemodynamic stability were included. We collected the following pre-VCE data from the patients: sex; age; co-morbidity; anticoagulant use, including aspirin, warfarin, and nonsteroidal anti-inflammatory drugs (NSAIDs); duration of hospital stay owing to overt OGIB; type of small-bowel preparation; hemoglobin level; and amount of blood transfusion. Specific therapeutic interventions were performed on the basis of VCE findings, including therapeutic colonoscopy, esophagogastroduodenoscopy, deep enteroscopy, interventional radiology, and surgical procedures.

The patients were divided into two groups: those who underwent VCE within 2 days of the last overt OGIB ( $<48$-h group) and those who underwent VCE more than 2 days after the last overt OGIB (>48-h group). This selection was established through a preliminary analysis of our records and review of previous studies. We compared the diagnostic yield, therapeutic intervention rate, length of hospital stay, and bleeding recurrence between the groups. This study was approved by the institutional review board of our institution (IRB No.ED 14073).

\section{Video capsule endoscopy}

VCE (PillCam SB and SB2, Given Imaging Ltd, Yokneam, Israel) was performed at bedside or outpatient clinic. Before the procedure, 2 or $4 \mathrm{~L}$ of polyethylene glycol was administered for smallbowel preparation. VCE images were reviewed by experienced gastroenterologists (K.B. and K.Y.S.) within 12-24 hours after the completion of the procedure using RAPID version 6 software (Given Imaging Ltd). Images were reviewed at a rate of 8-20 images per second. VCE findings were classified as either significant
Table 1 Characteristics of patients who underwent video capsule endoscopy for overt obscure gastrointestinal bleeding.

\begin{tabular}{|lll|}
\hline & Inpatients & Outpatients \\
\hline Capsule endoscopy, $\mathrm{n}$ & 79 & 15 \\
\hline Age, mean \pm SD, years & $54.5 \pm 18.7$ & $49.1 \pm 21.4$ \\
\hline Sex, male/female & $49 / 30$ & $12 / 3$ \\
\hline Anticoagulant, $\mathrm{n}$ & 6 & 1 \\
\hline NSAIDs, $\mathrm{n}$ & 4 & 1 \\
\hline Melena, $\mathrm{n}$ & 41 & 3 \\
\hline Hematochezia, $\mathrm{n}$ & 38 & 12 \\
\hline
\end{tabular}

SD, standard deviation; NSAIDs, nonsteroidal anti-inflammatory drugs.

or non-significant. If lesions were the possible source of bleeding, they were considered clinically significant. This includes active bleeding, angiodysplasia, ulcer, diverticulum (with stigmata of recent bleeding), small-bowel tumor, or bleeding outside the small bowel (i.e. stomach or colon). Blood clots were considered to be a positive finding. Lesions such as venous ectasia, erythematous mucosa, erosion, enteritis, and small non-bleeding polyps were considered to be non-significant findings.

\section{Statistical analysis}

Descriptive statistics were calculated to summarize the demographic and baseline characteristics of the patients. Traditional analytical statistics such as means, standard deviation, and percentages were used. VCE findings categorized by time group were analyzed using the $\chi^{2}$ test, Fisher exact test, or $t$ test where appropriate. In all tests, the level of significance was set at $P<$ 0.05. All statistical analyses were performed using SPSS version 18.0 (SPSS Inc., Chicago, IL, United States).

\section{Results}

\section{$\nabla$}

\section{Patient characteristics}

A total of 112 patients underwent VCE for overt OGIB. After excluding 18 patients lacking medical records, a total of 94 patients who underwent VCE after a negative findings on bidirectional endoscopy were analyzed ( $\bullet$ Fig. 1 ). The demographic characteristics of the patients are shown in Table 1. Twelve patients (12.7\%) were regularly consuming oral anticoagulant agents or NSAIDs. Melena and hematochezia were observed in $44(46.8 \%)$ and 50 patients ( $53.2 \%$ ), respectively. The mean minimum hemoglobin concentration was $9.49 \pm 2.26 \mathrm{~g} / \mathrm{dL}$, and the mean amount of blood transfusion before VCE was $1.82 \pm 1.87$ units.

The median duration between onset of overt OGIB and VCE was 4 days (range: 1 - 50 days).

The VCE findings are presented in Table 2 . The positive diagnostic yields of VCE among inpatients and outpatients were $53.2 \%$ and $33.3 \%$, respectively, but the difference was not statistically significant $(P=0.06)$. The overall positive yield of VCE was $48.9 \%$ (46 cases); these cases were predominantly active bleeding and angiodysplasia (19/46, 41.3\%).

Regarding the baseline characteristics and co-morbidity status of the $<48$-h and $>48$-h groups, there was no statistically significant difference with respect to anticoagulant or NSAID use. Moreover, there were no statistically significant differences in the minimum hemoglobin level or amount of blood transfusion. The incidence rates of diabetes mellitus, renal disease, hypertension, and liver cirrhosis were also comparable between the two groups. 
Table 2 Video capsule endoscopy findings.

\begin{tabular}{|c|c|c|c|}
\hline & $\begin{array}{l}<48-h \\
\text { group } \\
(n=30)\end{array}$ & $\begin{array}{l}>48-h \\
\text { group } \\
(n=64)\end{array}$ & $P$ value \\
\hline Positive finding, $\mathrm{n}(\%)$ & $20(66.7)$ & $26(40.6)$ & 0.019 \\
\hline Active bleeding, n (\%) & $6(20)$ & $1(1.6)$ & \\
\hline Angiodysplasia, n (\%) & $4(13.3)$ & $8(12.5)$ & \\
\hline Ulcer, n (\%) & $6(20)$ & $11(17.2)$ & \\
\hline Diverticulum, n (\%) & $1(3.3)$ & $2(3.1)$ & \\
\hline Tumor, n (\%) & $3(10)$ & $2(3.1)$ & \\
\hline Non-small-bowel lesion, n (\%) & 0 & $2(3.1)$ & \\
\hline
\end{tabular}

Table 3 Video capsule endoscopy outcomes.

\begin{tabular}{|llll|} 
& $\begin{array}{l}<48-\mathbf{h} \\
\text { group }\end{array}$ & $\begin{array}{l}\mathbf{> 4 8 - h} \\
\text { group }\end{array}$ & P value \\
\hline $\begin{array}{l}\text { Therapeutic intervention rate, } \\
\mathrm{n}(\%)\end{array}$ & $8(26.7)$ & $6(9.4)$ & 0.028 \\
\hline Rebleeding rate, $\mathrm{n}(\%)$ & $3(10)$ & $7(10.9)$ & 0.60 \\
\hline
\end{tabular}

The diagnostic yields of VCE to detect the cause of bleeding in the $<48$-h and > 48-h groups were 66.7\% (20/30) and 40.6\% (26/64), respectively ( $P=0.019 ; 0$ Table 2$)$.

We analyzed the rates of active bleeding and angiodysplasia lesions because of their clinical significance as indications for therapeutic intervention. Active bleeding was observed in $20 \%(6 / 30)$ and $1.6 \%(1 / 64)$ of the patients in the $<48$-h and $>48$-h groups, respectively. Angiodysplasia was observed in $13.3 \%(4 / 30)$ and $12.5 \%(8 / 64)$ of the patients in the $<48$-h and $>48$-h groups, respectively. The diagnostic yields for active bleeding and/or angiodysplasia were $33.3 \%$ and $14.1 \%$ in the $<48$-h and $>48-\mathrm{h}$ groups, respectively $(P=0.030)$. The diagnostic yields for detecting active bleeding and/or angiodysplasia decreased as time elapsed after the last overt OGIB ( $\bullet$ Fig. 2). The decrease in diagnostic yield with time in the $>48$-h group was strengthened by the lower rates of active bleeding and/or angiodysplasia in the $>48$-h group.

The diagnostic yield of VCE was $33.3 \%$ in the outpatient group, which is similar to that in the $>48$-h group. Furthermore, the diagnostic yield for active bleeding and/or angiodysplasia in the outpatient cohort was $13.3 \%$, which is comparable to that in the $>48$-h group (14.1\%).

Specific therapeutic interventions were performed in $26.7 \%$ (8/30) of the patients in the <48-h group: 1 therapeutic colonoscopy for clipping of angiodysplasia, 1 therapeutic esophagogastroduodenoscopy with ulcer coagulation, 2 radiologic interventions including selective embolization on active bleeding, and 4 surgical interventions for small-bowel tumors $(\mathrm{n}=2)$ or Meckel's diverticulum $(n=2)$. In comparison, only $9.4 \%(6 / 64)$ of the patients in the $>48$-h group underwent therapeutic intervention $(P=0.028$; $\odot$ Table 3$)$; interventions included surgical intervention for a small-bowel tumor $(n=1)$, ileal ulcer $(n=4)$, and jejunal angiodysplasia $(n=1)$. Three capsules were retained in the small bowel, one of which required surgical intervention.

The length of hospital stay was analyzed using the inpatient data. It decreased significantly at 5 days in the $<48$-h group (95\% confidence interval $[\mathrm{CI}], 4.8-7.7$ ) and at 7 days in the $>48$-h group (95\%Cl, 6.9-10.1; $P=0.039$; 0 Fig. 3). The mean amount of blood transfusion did not differ significantly between the groups ( $2.17 \pm$ 1.89 vs. $1.66 \pm 1.85$ units in the $<48$ - $h$ and $>48$-h groups, respec-

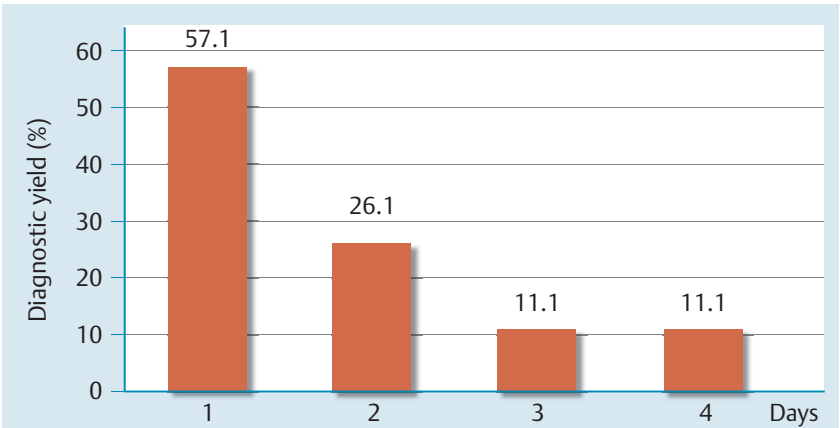

Fig.2 Diagnostic yield (\%) for detecting active bleeding and/or angiodysplasia with respect to the time (days) after the last gastrointestinal bleeding.

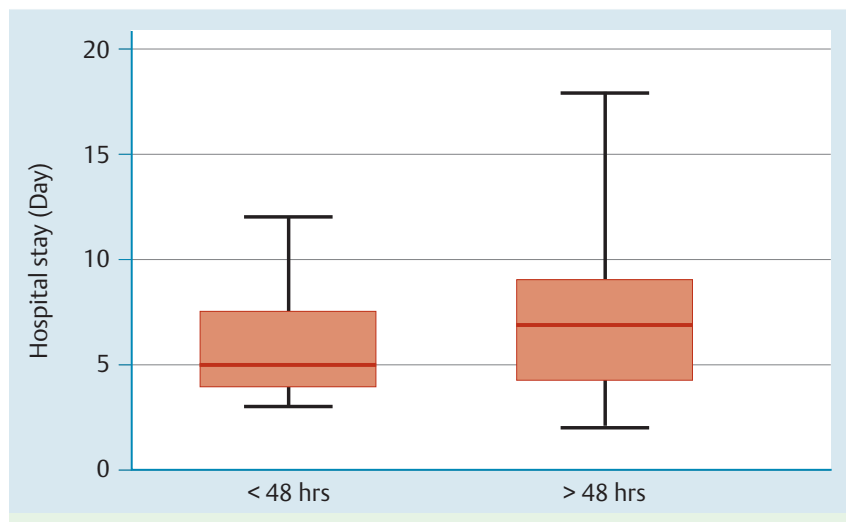

Fig. 3 Length of hospital stay (days) for the $<48$-h and $>48$-h groups.

tively; $P=0.23$ ). These results show that the severity of overt OGIB in both groups did not affect the therapeutic intervention rate or length of hospital stay.

During follow-up after VCE placement, 10 patients were hospitalized owing to rebleeding (10.6\%). The rebleeding rate did not differ significantly between the two groups $(P=0.60$; $\odot$ Table 3 ). The VCE findings of these patients included angiodysplasia $(\mathrm{n}=$ $4)$, ulcer $(n=2)$, tumor $(n=1)$, active bleeding without definite lesion $(n=1)$, and normal $(n=2)$. During the initial visit for overt OGIB, one of these patients was treated with endoscopic clipping, and surgical intervention was performed in another patient. However, the other eight patients did not receive therapeutic interventions.

\section{Discussion \\ $\nabla$}

VCE performed within 2 days of the last overt OGIB resulted in a higher diagnostic yield (66.7\%). In particular, the diagnostic yields for active bleeding and/or angiodysplasia were significantly higher in the $<48$-h group than in the $>48$-h group. Although this result is in line with previous studies $[3,4]$, there is no report about the efficacy of VCE application with a 48-h cutoff performed after normal bidirectional endoscopy in overt OGIB patients with hemodynamic stability.

VCE is an advanced technique for directly imaging the gastrointestinal tract [16]. Its high diagnostic yield and relatively low invasiveness make it an attractive diagnostic option for patients with OGIB $[9,17,18]$. Although the diagnostic yield of VCE ranges from $38 \%$ to $83 \%$ in OGIB [8], and early VCE examination may result in higher diagnostic yield $[3,4]$, the optimal timing for VCE 
deployment in overt OGIB patients remains controversial. In addition, there are no clear guidelines for, or mention of, the appropriate timing of VCE application in overt OGIB in the literature.

The high diagnostic yield of VCE performed within 2 days from the last overt OGIB in the present study corroborates the findings of previous studies performed in patients with overt OGIB. Singh et al. [14] reported a higher overall diagnostic yield when VCE was performed within the first 3 days after the onset of overt OGIB; they found that the yield to identify active bleeding gradually decreased each day after hospitalization. However, it is noteworthy that the diagnostic yield could be better when VCE is applied earlier than 3 days after overt OGIB with regard to bleeding sequence, which has a trend to naturally stop with time.

The present findings show that VCE performed within 2 days of the last OGIB (i.e. closer to onset) enabled physicians to achieve higher diagnostic yields. This result is corroborated by Yamada et al. [3], who investigated 90 patients with overt OGIB; in their study, VCE determined the origin of bleeding in 46 patients (51\%), with diagnostic yields of $73 \%, 48 \%, 50 \%$, and $35 \%$ in the first, second, third, and fourth quartiles, respectively. These results indicate a need to simplify diagnostic work-up and perform VCE earlier in overt OGIB patients.

It is often difficult to perform VCE strictly within 2 days in patients with overt OGIB occurrence. In the present study, the time to VCE varied greatly ( 1 - 50 days). There are several possible reasons for the time delay of VCE, such as protracted observation at an outside clinic or hospital, intermittent occurrence of overt OGIB symptoms, and various diagnostic procedures before VCE. However, taking these results into account, the time delay could make it more difficult to define the bleeding lesion in patients with overt OGIB.

Small-bowel preparation was usually performed with 2 or $4 \mathrm{~L}$ of polyethylene glycol. When VCE was performed 1 day after the last overt OGIB occurrence, it could be successfully performed 2 $\mathrm{h}$ after polyethylene glycol administration. If bowel preparation for total colonoscopy had already been performed for the evaluation of bleeding, further bowel preparation was unnecessary. Similar to previous studies $[3,14,15]$, the present study revealed a significantly greater rate of specific therapeutic interventions in the < 48-h VCE group. VCE deployment within 2 days of the last overt OGIB after the patient had undergone bidirectional endoscopy with a negative result could afford a higher diagnostic yield and allow for the immediate administration of appropriate therapy. This would consequently improve treatment outcomes in overt OGIB patients. Regarding the length of hospital stay, this was approximately $28.6 \%$ shorter in the $<48$-h VCE group. This time-related benefit of deploying VCE within 2 days of the last OGIB could save a substantial amount of national healthcare resources.

Our study showed that rebleeding occurred in 10 patients after the initial visit. Four of them presented angiodysplasias, which have been shown to be predictive factors of unfavorable clinical outcome [19]. Regarding the patients who showed rebleeding, angiodysplasia occurred in $66.7 \%$ and $14.3 \%$ of patients in the $<48$-h and >48-h groups, respectively. Angiodysplasia often presents a multifocal character and a significant rebleeding rate, even when these patients are treated endoscopically [20,21].

The present study has some limitations. First, it has a retrospective design. However, we enrolled all consecutive patients with overt OGIB who received VCE in our hospital. In addition, the treatment process was standardized for all patients with overt
OGIB in our gastrointestinal bleeding unit. These factors collectively reduce the drawbacks of the retrospective study design. In conclusion, VCE performed within 2 days of the last overt OGIB after a negative bidirectional endoscopic finding achieves a high diagnostic yield in $66.7 \%$ of patients, leading to therapeutic intervention in $26.7 \%$ as well as reducing the length of hospital stay by $28.6 \%$. These results indicate that VCE may play a crucial diagnostic role when performed close (i.e. $<2$ days) to the onset of overt OGIB. Performing VCE within 2 days could improve the management of these patients by allowing for a more rapid, appropriate, and cost-effective therapeutic plan.

\section{Competing interests: None}

\section{Acknowledgments}

$\nabla$

This work was supported by the National Research Foundation of Korea (NRF) grant funded by the Korean Government (NRF2014R1A2A2A01006131) and Grant of Korea University.

\section{References}

1 Zuckerman GR, Prakash C, Askin MP et al. AGA technical review on the evaluation and management of occult and obscure gastrointestinal bleeding. Gastroenterology 2000; 118: $201-221$

2 Leighton JA, Goldstein J, Hirota W et al. Obscure gastrointestinal bleeding. Gastrointest Endosc 2003; 58: 650-655

3 Yamada A, Watabe H, Kobayashi $Y$ et al. Timing of capsule endoscopy influences the diagnosis and outcome in obscure-overt gastrointestinal bleeding. Hepatogastroenterology 2012; 59: 676-679

4 Bresci $G$, Parisi $G$, Bertoni $M$ et al. The role of video capsule endoscopy for evaluating obscure gastrointestinal bleeding: usefulness of early use. J Gastroenterol 2005; 40: 256-259

5 Laine L, Sahota A, Shah A. Does capsule endoscopy improve outcomes in obscure gastrointestinal bleeding? Randomized trial versus dedicated small bowel radiography Gastroenterology 2010; 138: 1673 - 1680

6 Pennazio M, Santucci R, Rondonotti E et al. Outcome of patients with obscure gastrointestinal bleeding after capsule endoscopy: report of 100 consecutive cases. Gastroenterology 2004; 126: 643 -653

7 Liao Z, Gao R, Xu C et al. Indications and detection, completion, and retention rates of small-bowel capsule endoscopy: a systematic review. Gastrointest Endosc 2010; 71: 280-286

8 Rondonotti E, Villa F, Mulder CJ et al. Small bowel capsule endoscopy in 2007: indications, risks and limitations. World J Gastroenterol 2007; 13: $6140-6149$

9 Pennazio M, Eisen G, Goldfarb N et al. ICCE consensus for obscure gastrointestinal bleeding. Endoscopy 2005; 37: 1046-1050

10 Triester SL, Leighton JA, Leontiadis GI et al. A meta-analysis of the yield of capsule endoscopy compared to other diagnostic modalities in patients with obscure gastrointestinal bleeding. Am J Gastroenterol 2005; 100: 2407-2418

11 Leighton JA, Triester SL, Sharma VK. Capsule endoscopy: a meta-analysis for use with obscure gastrointestinal bleeding and Crohn's disease. Gastrointest Endosc Clin N Am 2006; 16: 229-250

12 Hadithi M, Heine GD, Jacobs MA et al. A prospective study comparing video capsule endoscopy with double-balloon enteroscopy in patients with obscure gastrointestinal bleeding. Am J Gastroenterol 2006; 101: $52-57$

13 Almeida N, Figueiredo P, Lopes $S$ et al. Urgent capsule endoscopy is useful in severe obscure-overt gastrointestinal bleeding. Digest Endosc 2009; $21: 87-92$

14 Singh A, Marshall C, Chaudhuri B et al. Timing of video capsule endoscopy relative to overt obscure GI bleeding: implications from a retrospective study. Gastrointest Endosc 2013; 77: 761 - 766

15 Lecleire S, Iwanicki-Caron I et al. Yield and impact of emergency capsule enteroscopy in severe obscure-overt gastrointestinal bleeding. Endoscopy 2012; 44: 337-342

16 Iddan G, Meron G, Glukhovsky A et al. Wireless capsule endoscopy. Nature 2000; 405: 417 
17 ASGE Standards of Practice Committee. Fisher L, Lee KrinskyM et al. The role of endoscopy in the management of obscure GI bleeding. Gastrointest Endosc 2010; 72: 471 - 479

18 Rondonotti E, Marmo R, Petracchini $M$ et al. The American Society for Gastrointestinal Endoscopy (ASGE) diagnostic algorithm for obscure gastrointestinal bleeding: eight burning questions from everyday clinical practice. Dig Liver Dis 2013; 45: 179-185
19 Hindrycks $P$, Botelberge T, De Vos $M$ et al. Clinical impact of capsule endoscopy on further strategy and long-term clinical outcome in patients with obscure bleeding. Gastrointest Endosc 2008; 68: 98-104

20 Richter JM, Christensen MR, Colditz GA et al. Angiodysplasia: natural history and efficacy of therapeutic interventions. Dig Dis Sci 1989; 34: $1542-1546$

21 Saurin JC, Delvaux M, Vahedi K et al. Clinical impact of capsule endoscopy compared to push enteroscopy: 1-year follow-up study. Endoscopy $2005 ; 37: 318-323$ 